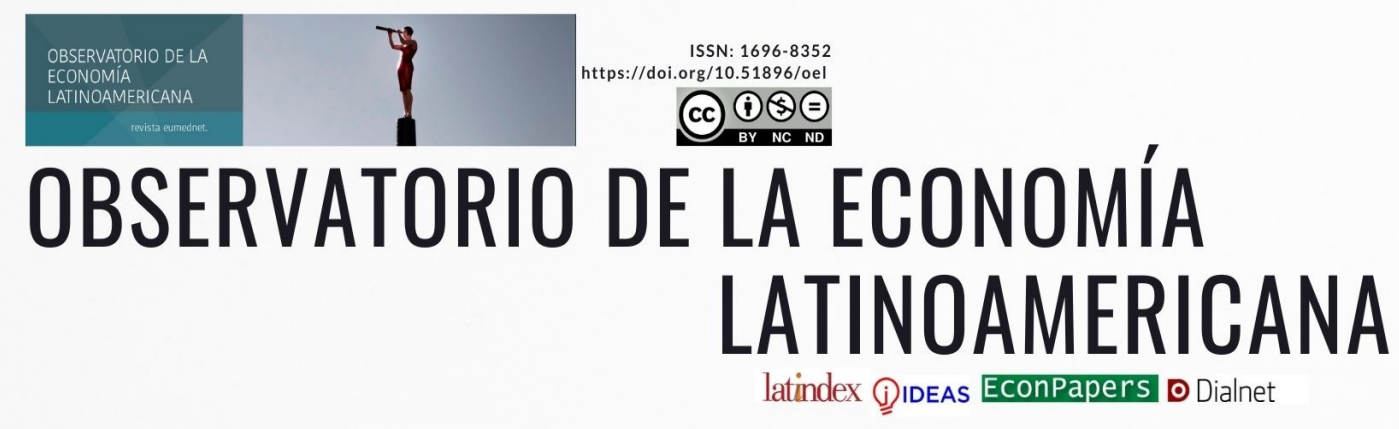

\title{
GESTION POR PROCESOS E INDICADORES DE CUMPLIMIENTO EN LA CONTRATACIÓN PÚBLICA
}

\author{
Ing. Evelin Magdalena Alcívar Chóez \\ Facultad de Administración \\ Universidad Laica Vicente Rocafuerte de Guayaquil \\ ealcivarc@ulvr.edu.ec
}

MAE. Verónica Violeta Rodríguez Basantes

Docente de la Facultad de Administración

Universidad Laica Vicente Rocafuerte de Guayaquil

vrodriguezb@ulvr.edu.ec

Para citar este artículo puede utilizar el siguiente formato:

Evelin Magdalena Alcívar Chóez y Verónica Violeta Rodríguez Basantes: "Gestion por procesos e indicadores de cumplimiento en la contratación pública.", Revista Observatorio de la Economía Latinomericana (Vol 19, № 3, pp. 80-95, marzo 2021). En línea:

https://www.eumed.net/es/revistas/economia-latinoamericana/oel-marzo21/contratacion-publica

\section{RESUMEN}

En el Ecuador la contratación pública se encuentra regida por la Ley Orgánica del Sistema Nacional de Contratación Pública (LOSNCP) y su Reglamento. El objeto de la presente investigación consiste en examinar cómo se han venido dando las contrataciones públicas del sector salud en el Ecuador y los aspectos relacionado a la problemática que se presentan en Las Direcciones Distritales de Salud por la presencia de una gestión por proceso inadecuada, por tanto, se definieron objetivos que permitieron el direccionamiento de la investigación. Se estableció los aspectos teóricos que se utilizaron para fundamentar el análisis de la eficiencia y eficacia de los procesos, la identificación de amenazas y oportunidades que se presenta durante la gestión de una organización, permitiendo identificar las mejores prácticas que permitieron establecer inferencias sólidas y una solución para mejorar las falencias dentro del proceso de contratación pública ejecutado por los ministerios de salud pública. La presente investigación se desenvolverá mediante un enfoque cualitativo. La técnica para usar es la triangulación de datos, a través de observación a los procesos, encuestas a los involucrados directos y revisión de la documentación proporcionada por las Direcciones Distritales de Salud de Manabí. El artículo concluye con las respectivas conclusiones y recomendaciones para los 
procesos desempeñados durante el proceso de contratación pública de los Ministerios de Salud Pública.

Palabras claves: Gestión por Procesos, Indicadores de productividad, calidad, administración pública.

\section{MANAGEMENT BY PROCESSES AND INDICATORS OF COMPLIANCE IN PUBLIC PROCUREMENT}

\section{ABSTRACT}

In Ecuador, public procurement is governed by the Organic Law of the National Public Procurement System (LOSNCP) and its Regulations. The purpose of this research is to examine how the public procurement of the health sector has been taking place in Ecuador and the aspects related to the problem that arise in the District Health Directorates in the presence of an inadequate management of the process, therefore, Objectives were defined that allowed the direction of the investigation. The theoretical aspects that were used to base the analysis of the efficiency and effectiveness of the processes, the identification of threats and opportunities that arise during the management of an organization, were established, allowing to identify the best practices that allowed to establish solid inferences and a solution for improve deficiencies within the public procurement process carried out by the ministries of public health. This research will be developed through a qualitative approach. The technique to be used is the triangulation of data, through the observation of the processes, surveys of those directly involved and review of the documentation provided by the District Health Directorates of Manabí. The article concludes with the respective conclusions and recommendations for the processes carried out during the public procurement process of the Ministries of Public Health.

Keywords: Process management, Indicators of productivity, quality, public administration.

\section{1.- INTRODUCCIÓN}

La gestión por procesos como herramienta que permite mantener la mejora continua en todo tipo de organización, aumentando la eficiencia y eficacia de los diferentes procesos que las integran. Además, permite controlar el cumplimiento de objetivos, metas, políticas, entre otras. Asimismo, estas se apoyan con indicadores de gestión, cumplimiento, financieras y demás, para basar su análisis y establecer sus soluciones basadas en resultados.

\section{2.- Planteamiento del Problema}

A nivel mundial, para las entidades del sector público el contar con un proceso efectivo de compras públicas garantiza el manejo apropiado de los recursos designados, la transparencia en la gestión de adquisición de bienes o servicios, la libre competencia y el acceso a nuevas tecnologías 
que promueven la gestión de licitación con apego a las leyes y reglamentos de contrataciones públicas.

El entorno de compras públicas para América Latina y el Caribe presentó un rezago en comparación con otras experiencias de integración regional, especialmente la europea; sin embargo, la existencia de instrumentos e iniciativas internacionales, aportan un punto de partida para emprender mejoras, a nivel normativo y administrativo, en los sistemas nacionales de compras públicas de la región. Para asegurar el éxito de las contrataciones públicas, estas deben ser transparentes y eficientes dando como resultado un impacto positivo en las condiciones de gobierno y en la percepción que los ciudadanos tienen sobre su régimen de gobierno (Organización Mundial del Comercio, 2016).

La contratación pública en el Ecuador ha evolucionado vertiginosamente, desde la promulgación de la Ley Orgánica del Sistema Nacional de Contratación Pública. En la parte técnica, la Ley ha creado una institución exclusiva para dirigir, elaborar políticas, ejecutarlas y controlar todo el sistema de la contratación pública (Altamirano, 2014).

No obstante, se han presentados distintos casos de sobornos y de corrupción productos de las falencias presentadas durante el proceso de compras públicas al incumplir con los criterios y requisitos establecidos en las leyes, reglamentos, normas, entre otras métricas que facultan una apropiada gestión por proceso en las entidades públicas del Ecuador.

De modo que, la presente investigación buscó mejorar la gestión por procesos de los Ministerios de Salud Pública y velar por el cumplimiento de los aspectos legales y normativos que regulan la gestión de contratación pública en éstos tipo de entidades. Dentro del campo de la medicina, algunos establecimientos públicos de salud a nivel país, han presentado diferentes problemas en la adquisición de bienes y servicios; con esto se corrobora la calidad de la atención médica que se presta en hospitales públicos y centros de salud con alta demanda de pacientes, que está sufriendo, desde hace tiempo, los efectos de los atrasos de compras públicas de insumos médicos, en especial cuando se trata de reparar en tiempo y forma aparatos que requieren de piezas o repuestos que sólo se consiguen a través de este proceso (Córdova, 2014).

A esto se suma la falta de médicos, el sistema público ecuatoriano de salud tiene un déficit de 6000 galenos a escala nacional. Las especialidades con más vacíos son: anestesiología, cardiología, endocrinología, pediatría y neurología, entre otras. (Soledispa, 2016).

De manera que, considerando la problemática existente en la gestión de compras pública la presente investigación se desarrolló en las Direcciones Distritales de Salud en Manabí.

\section{3.- Sistematización del problema}

¿Qué referentes teóricos respaldan la presente investigación? 
¿Cómo se debe diagnosticar las diferentes etapas de los procedimientos de contratación pública?

¿Cuáles son los factores críticos en los procesos de contratación pública de las Direcciones Distritales de Salud?

¿Cómo debe estar diseñado el modelo de gestión por procesos en la contratación pública que aporte al cumplimiento de los indicadores de las Direcciones Distritales de Salud?

\section{4.- Objetivos de la Investigación}

\section{1.- Objetivo general}

Diseñar un modelo de gestión por procesos que aporte al cumplimiento de los indicadores de Contratación Pública de las Direcciones Distritales de Salud.

\section{2.- Objetivos específicos}

- Establecer el marco teórico que respalden la presente investigación.

- Diagnosticar la eficiencia y eficacia del proceso de contratación pública.

- Identificar los factores críticos en los procesos de contratación pública de las Direcciones Distritales de Salud.

- Integrar métodos y buenas prácticas para la buena gestión por proceso de contratación pública en las Direcciones Distritales de Salud.

\section{5.- Justificación de la investigación}

\section{1.- Justificación Práctica}

El presente proyecto busca diseñar un modelo de gestión por procesos que aporte al cumplimiento de los indicadores de los procesos de Contratación Pública de bienes, obras y servicios de las Direcciones Distritales de Salud el cual se considera aplicable y justificable debido a los retrasos que se han presentado en las diferentes Casas de Salud y que al ponerlo en práctica garantizará una correcta gestión de compra, asegurando que la empresa tenga los mejores proveedores para abastecer los mejores productos y servicios, reducir los costos y optimizar el gasto público.

\section{2.- Justificación Metodológica}

La investigación tiene un enfoque cualitativo, en el cual se observará el proceso actual que realizan las Direcciones Distritales de salud en Manabí en los procesos de compra pública de bienes y servicios se analizarán las causas de la insatisfacción actual de los pacientes de esta área determinando las falencias en cada etapa del proceso de compra pública.

\section{3.- Justificación Social}


El diseño de estrategias para fortalecer la adquisición de bienes y servicios en las Direcciones Distritales de Salud en Manabí permitirá Incrementar la satisfacción de los ciudadanos con respecto a los servicios de salud en este sector de la población.

\section{4.- Justificación Económica}

Los procesos de diseño estrategias para fortalecer la adquisición de bienes y servicios en las Direcciones Distritales de Salud en Manabí ayudaran en la operatividad y al correcto funcionamiento de estas Entidades Estales a través de un óptimo control y direccionamiento en las áreas comprometidas. El personal percibirá un mejor ambiente laboral, desarrollando sus actividades con mayor productividad y buscarán la satisfacción del cliente.

Con la presente investigación se busca optimizar el gasto público y que sean ejecutados de acuerdo a las necesidades Institucionales enfocadas en el Plan Operativo Anual y Plan Anual de Contratación, garantizando la provisión de los servicios de salud con calidad y calidez.

\section{6.- Intencionalidad de la investigación}

\subsection{Delimitación del problema de investigación}

Delimitación Espacial: País Ecuador- Provincia de Manabí

Delimitación Temporal: 2019

Delimitación del Universo: Direcciones Distritales de Salud de Manabí.

Delimitación del contenido en el campo amplio del conocimiento: Administración

Delimitación del contenido en el campo específico del conocimiento: Gestión por Procesos, Indicadores de Cumplimiento, Calidad, Administración Pública.

\section{7.- Idea a defender}

Con el diseño de un modelo de gestión por procesos se contribuye significativamente al cumplimiento de los indicadores de las Direcciones Distritales de Salud

\section{8.- Fundamentación Teórica}

\section{1.- Teoría de la Administración}

Según Dessler (2009) señalo que: en el proceso de administración varios expertos coinciden en que hay cinco funciones básicas que todos los gerentes desempeñan: la planeación, la organización, la integración, la dirección y el control. Algunas de las actividades específicas de cada función son:

- $\quad$ Planeación. Establecer metas y normas; elaborar reglas y procedimientos; desarrollar planes y pronósticos. 
- $\quad$ Organización. Asignar una tarea específica a cada subordinado; establecer departamentos; delegar funciones en los subordinados; determinar canales de autoridad y comunicación; coordinar el trabajo de los subordinados.

- Integración. Determinar qué tipo de personal se debe contratar, reclutar a posibles empleados, seleccionarlos, establecer normas de desempeño para

- ellos, remunerarlos, evaluar su desempeño, asesorarlos, capacitarlos y hacer que se desarrollen.

- Dirección. Encargarse de que otros cumplan su trabajo, mantener un buen estado de ánimo y motivar a los subordinados.

- Control. Establecer normas como cuotas de ventas y estándares de calidad con niveles de producción; comparar el desempeño real con esos estándares, y tomar las medidas correctivas necesarias.

\section{2.- Gestión por Procesos}

Según (Perez, 2004) El concepto de gestión lleva asociada la idea de acción para que los objetivos fijados se cumplan. Un modelo para visualizar el proceso de gestión es el conocido, pero no por ello suficientemente aplicado, ciclo Deming o PDCA; es fundamental que la lógica que en el subyace sea comprendida y practicada por todos los mandos y directivos de la empresa. Un buen plan de acción garantiza en gran medida que los costes de los recursos (personas, materiales, etc.) se traducirán en valor (para la empresa y el cliente).

\section{Figura 1}

Ciclo Deming

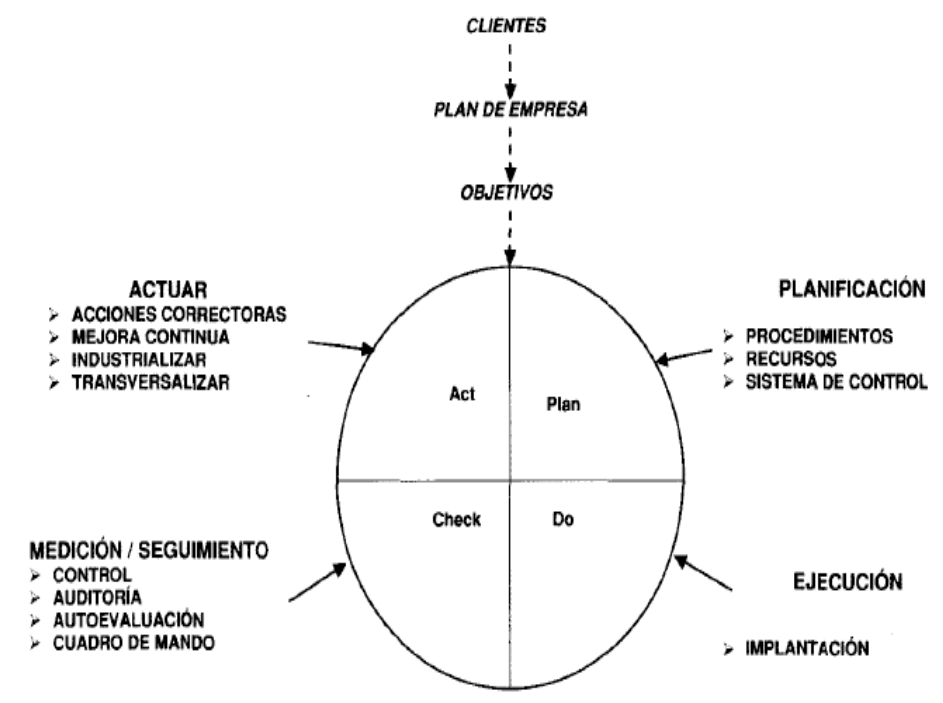

Fuente: (Perez, 2004) 


\section{3.- Indicadores de Gestión}

Según los indicadores de Gestión en cuanto a su naturaleza se clasifican según los factores clave de éxito. Los indicadores de gestión deben reflejar el comportamiento de algunos factores y actores, de esta forma se determina los indicadores de efectividad, de eficiencia y eficacia de la productividad.

Se detalla la interrelación de los diferentes factores.

\section{Figura 2}

Mapa de factores clave de éxito en la gestión

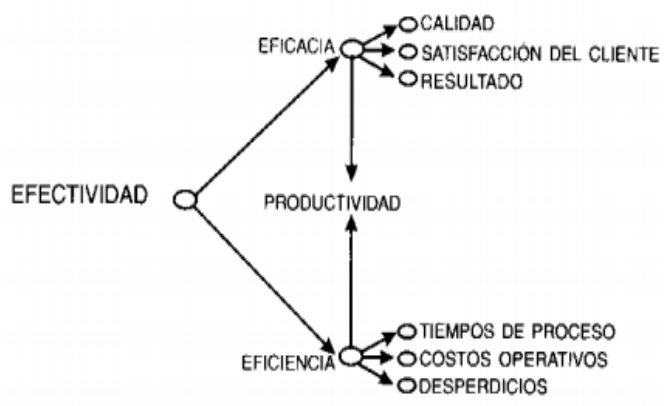

Fuente: (Beltrán, 2005)

La eficiencia se debe entender e identificar con la productividad de los recursos ya que equivale a la relación entre cantidad producida y recursos consumidos, y la eficacia comprendería el nivel de contribución al cumplimiento de los objetivos de la empresa por tanto la competitividad para ser eficaz hay que ser previamente eficiente, es por ello que la dificultad de su detección, está en hacer bien aquello que no se debería de estar haciendo mientras que la flexibilidad se asocia con la capacidad de adaptación a una situación específica, como procedimientos, descripción de los puestos de trabajo, estilos de dirección y estructura organizativa. Lo que enmarcan a la calidad en incorporar herramientas que potencien las capacidades individuales y de los grupos para mejorar la eficacia de su desempeño.

\section{9.- Tipo de Investigación}

El presente proyecto tiene un enfoque cualitativo. La investigación cualitativa proporciona profundidad a los datos, dispersión, riqueza interpretativa, contextualización del ambiente o entorno, detalles y experiencias únicas. Asimismo, aporta un punto de vista "fresco, natural y holístico" de los fenómenos, así como flexibilidad. (Hernández, Fernández, \& Baptista, 2014).

Esta perspectiva faculta a los investigadores a recopilar y trabajar con datos no numéricos y busca interpretar el significado de estos datos que permitirán comprender la vida laboral y social a través del estudio de poblaciones o lugares seleccionados. 
Se va utilizar como alcance de investigación descriptiva. Estudios descriptivos se analizan cualquier fenómeno y busca especificar propiedades y características importantes. Describe tendencias de un grupo o población, únicamente pretenden medir o recoger información de manera independiente o conjunta sobre los conceptos o las variables a las que se refieren. (Hernández, Fernández, \& Baptista, 2014).

La investigación descriptiva permite analizar de forma detallada y precisa cada información que se obtenga sobre las deficiencias de los procesos de Contratación Pública de las Direcciones Distritales de Salud. Se desarrollará bajo un diseño: No experimental, Transversal.

Según Hernández, Fernández, \& Baptista, (2014) La investigación no experimental son estudios que se realizan sin la manipulación deliberada de variables y en los que sólo se observan los fenómenos en su ambiente natural para analizarlos.

Diseños transeccionales (transversales) Forma parte de la clasificación del diseño no experimental y se centra en recopilar datos en un momento único. Su propósito es describir variables y analizar su incidencia e interrelación en un momento dado.

La investigación se considera como no experimental, ya que se van a analizar datos reales, tal y como ha ocurrido en su contexto natural, como lo es el incumplimiento de los indicadores en los procesos de contratación pública de la Direcciones Distritales de Salud.; así como también tiene un diseño de investigación transversal ya que se recopilan datos una única vez en un momento determinado.

\section{0.- Población}

Según Lerma (2014) señaló que: "La población está conformada por un conjunto de elementos (personas, animales, compañías, entre otras) que poseen y comparten características similares referente a la problemática existente, en la que se establece su dimensión y límites geográficos al interior de la misma”. (p. 105)

Por tanto, la población seccionada son los funcionarios públicos relacionados directamente en el proceso de contratación pública de las Direcciones Distritales de Salud.

\section{1.- Muestra}

$>$ No Probabilística

$>$ A Conveniencia

$>$ Casos de Estudio: 3

Muestra no probabilística o dirigida a Subgrupo de la población en la que la elección de los elementos no depende de la probabilidad, sino de las características de la investigación, se basa en 
la documentación de ciertas experiencias. Muestra por Conveniencia están formadas por los casos disponibles a los cuales tenemos acceso. (Hernández, Fernández, \& Baptista, 2014).

Para la presente investigación se considerará al personal que interviene en las diferentes etapas de los procesos de Contratación Pública de las Direcciones Distritales de Salud de la Provincia de Manabí, como son:

$>$ Analista Distrital de Planificación

$>$ Analista de Adquisiciones

> Analista Distrital de Presupuesto y Administración de Caja

$>$ Analista Distrital Administrativo Financiero

\section{2.- Instrumento de investigación.}

La técnica a ser usada en la investigación es la triangulación cualitativa:

$>$ Entrevista

$>$ Observación

$>$ Revisión Documental

Según Hernández, Fernández, \& Baptista, (2014). Observación cualitativa implica profundizar en situaciones sociales y mantener un papel activo, estar atento a los detalles, sucesos, eventos e interacciones, así como una reflexión permanente. Un buen observador necesita saber escuchar y utilizar todos los sentidos, poner atención a los detalles, poseer habilidades para descifrar y comprender conductas, ser reflexivo y flexible para cambiar el centro de atención, si es necesario.

Entrevista se define como una reunión para conversar e intercambiar información entre una persona (el entrevistador) y otra (el entrevistado) u otras (entrevistados). En el último caso podría ser tal vez una pareja o un grupo pequeño como una familia $o$ un equipo de manufactura. En la entrevista, a través de las preguntas y respuestas se logra una comunicación y la construcción conjunta de significados respecto a un tema.

Revisión Documental es una fuente muy valiosa de datos cualitativos nos pueden ayudar a entender el fenómeno central de estudio. Prácticamente la mayoría de las personas, grupos, organizaciones, comunidades y sociedades los producen y narran, o delinean sus historias y estatus actuales. Le sirven al investigador para conocer los antecedentes de un ambiente, así como las vivencias o situaciones que se producen en él y su funcionamiento cotidiano y anormal.

En la presente investigación se usará la técnica de triangulación cualitativa de datos, debido a que con lo citado anteriormente, nos permitirá profundizar más en este tema de estudio en la obtención de datos en las Direcciones Distritales de Salud, con la observación, entrevista a expertos en el tema y documentación se analizará la información obtenida la que servirá para determinar los factores críticos en los procesos de contratación pública de las Direcciones Distritales de Salud, que 
será esencial para diseñar un modelo de gestión que permita mejorar los indicadores de cumplimiento.

\section{3.- Presentación de Resultados}

Luego del trabajo de recolección de información relacionada a los procesos de contratación pública de los Distritos de Salud de Bolívar, Paján y Rocafuerte los resultados finales fueron:

\section{Resultados de la Observación}

- Para los procesos de contratación pública, los Distritos aplican la normativa actual vigente establecida por el SERCOP y procedimientos internos del MSP.

- De manera obligatoria utilizan las herramientas informáticas tanto para los Procedimientos Dinámicos como para el Régimen Común.

- Para iniciar los procesos de contratación es necesario que el área requirente inicie el proceso con la documentación preparatoria que sustente la necesidad, de aquí parte que se dé el cumplimiento de los indicadores de gestión.

- Los responsables de realizar el monitoreo, seguimiento y control a las compras no ejecutadas del PAC en el periodo programado son los analistas del Departamento Administrativo Financiero, el área de Planificación y los Administradores de los procesos de las áreas requirentes.

- Los factores que inciden en el logro de los objetivos de gestión procesos de contratación pública son el trabajo en equipo, la responsabilidad y la ética de los servidores públicos que laboran en las áreas requirentes, los administradores de contratos y órdenes, y demás analistas. Además, es importante la selección del proveedor, ya que este debe cumplir con los compromisos adquiridos y la oportuna entrega - recepción de bienes, servicios u obras requeridos.

- La efectividad de los procesos de compras se mide con la ejecución presupuestaria y los resultados que arrojen los indicadores de gestión, evaluación que se realiza a través del monitoreo cuatrimestral del PAC.

- Para cumplir los procesos de contratación el área requirente debe identificar la necesidad de compra, y preparar las especificaciones técnicas y/o términos de referencia, y el cálculo del presupuesto referencial, certificación presupuestaria y certificación del PAC, y quienes hagan las veces de administrador de contrato y comisión de recepción deben velar por el cumplimiento de las obligaciones que establece la normativa.

- El cumplimiento de los indicadores de gestión se comprueba con la ejecución del PAC cuatrimestral y el presupuesto devengado; además de la revisión que se realiza a los documentos de los procesos de contratación.

- Los controles que se realizan en los procesos de adquisición inician con la revisión del PAC, ya que el proceso debe constar en la planificación del Distrito, el responsable del área Administrativa debe realizar una verificación de la documentación del proceso precontractual, 
la misma que debe estar completa desde el inicio que debe incluir los estudios y diseños completos, definitivos y actualizados, planos y cálculos, Términos de Referencia y Especificaciones Técnicas (TDR/ET), realizar verificaciones en el catálogo electrónico, entre otros. Otro control que se realiza es el seguimiento cuatrimestral al PAC.

- Los indicadores que se aplican permiten medir la eficiencia y eficacia de los procesos de contratación.

- Los resultados de los indicadores ayudan a plantear medidas correctivas y decisiones futuras en la gestión de cada Distrito. Permiten mejorar la ejecución de los procesos de contratación y agilidad en el flujo de información.

- Para evaluar la efectividad de la gestión por procesos en la contratación pública en los Distritos se requiere tener manuales de procedimientos ya sea de manera general o por tipo de contratación, que especifique las actividades para cada área responsable.

- Es necesario capacitar al personal en materia de contratación pública.

- Se debe socializar la normativa de Contratación Pública, Código Orgánico de Finanzas Públicas y demás normativa, y formatos que se utilizan en el proceso de contratación para trabajar de manera coordinada interdepartamentalmente.

- Asesorar siempre que se necesite o se solicite, sobre todo a las áreas requirentes.

- Motivar a las áreas el cumplimiento de la Planificación Anual de Contrataciones de cada Entidad.

- Los indicadores que se aplican en las fases del proceso de contratación pública de los distritos son:

Bolívar:

"Revisión TDR/ET, Órdenes de compra generadas, Contratos registrados oportunamente."

Paján:

"Los indicadores mensuales que se aplican en las fases de los procesos de Contratación Pública son:

- Número de contratos registrados en el portal / Número de contratos recibidos

- Número de términos de referencia y/o especificaciones técnicas revisados oportunamente / Número de términos de referencia y/o especificaciones técnicas recibidos."

Rocafuerte:

"Índice de órdenes de compras emitidas, contratos registrados en el Sistema Oficial de Contratación Pública del Ecuador (SOCE) y Especificación Técnicas o Términos de referencia revisados"

\section{Resultados de las encuestas}

- El $67 \%$ de los encuestados mencionaron que tienen más de 6 años laborando en los Distritos de Salud seleccionados.

- Todos los encuestados respondieron que están "De acuerdo" que las funciones y procedimientos de contratación pública establecida como parte de la gestión de procesos se alinea a su realidad. $Y$ que cumplen con la normativa vigente. Indicaron que es necesario 
realizar evaluaciones a las funciones y procedimientos que permitan mejorar la eficiencia y eficacia en lo relacionado a la contratación pública.

- El $67 \%$ de los encuestados indicaron que siempre se ejecutan las compras programadas en el PAC en el periodo establecido, mientras que el $33 \%$ indicó que a veces.

- Más del $50 \%$ de los encuestados mencionó que nunca ha recibido capacitación para el óptimo desempeño de sus funciones, y quienes contestaron que sí habían recibido capacitación fue el 33\%, e indicaron que la capacitación recibida fue anual.

- El $67 \%$ de los encuestados mencionaron que si se ha establecido un tiempo para la ejecución de actividades y entrega de documentación entre los diferentes responsables del proceso de compra.

- El $67 \%$ de los encuestados respondieron entre las opciones que están "Muy de acuerdo" y "De acuerdo" que la aplicación de indicadores permite medir la eficiencia y eficacia en los procesos de compras públicas. Mientras que el $33 \%$ de los encuestados son indiferentes sobre la efectividad del uso de indicadores para medir la operación de los procesos de compras públicas.

- Los encuestados manifestaron estar de acuerdo en que es necesario realizar una evaluación a la estructura de los indicadores aplicados en el proceso de contratación pública.

- Los encuestados indicaron que es necesario diseñar, y por ende ejecutar, un modelo de gestión por proceso, ya que permitirá mejorar la eficiencia y eficacia de los procesos de contratación pública y al cumplimiento de los indicadores.

\section{Resultados de la revisión documental}

- El nivel de ejecución cuatrimestral en los Distritos es superior al $80 \%$

- Las actividades planificadas son ejecutadas en su mayoría o en su totalidad en el cuatrimestre que corresponde.

- La ejecución del PAC en el Distrito de Salud de Bolívar es alta, 100\% de ejecución, tanto en el segundo y tercer cuatrimestre se ejecutaron todas las actividades programadas en el PAC y se realizaron las respectivas adjudicaciones que se encontraban planificadas. Mientras que en el primer cuatrimestre no se cumplió sólo una actividad que correspondía a la contratación del servicio de envíos de correspondencia a un Hospital y el presupuesto fue retirado por parte de la Coordinación Zonal 4, el resultado del indicador fue del 96,67\%

- La ejecución del PAC en el Distrito de Salud de Paján fue en 84,12\% en el I cuatrimestre, $97,86 \%$ en el II cuatrimestre y $98,35 \%$ en el III cuatrimestre. La justificación que dio el Distrito de Salud de Paján en cuanto a la ejecución del $84,12 \%$ del PAC en el primer cuatrimestre, fue que 2 Procesos se declararon desiertos: 1 reprogramado y 1 pendiente. $Y$ que el material de ferretería no se ejecutó por cambio de personal Auxiliar de Mantenimiento. Y que ambas actividades se ejecutarían en el segundo cuatrimestre.

- El PAC del Distrito de Paján concentra la mayor parte de planificación de actividades los primeros cuatro meses del año y luego estas disminuyen en los cuatrimestres II y III a menos de la mitad de lo programado en el Cuatrimestre I. 
- Se realizó la revisión a los procedimientos de "Gestión Preparatoria y Precontractual" y "Gestión Contractual" que fueron aprobados en octubre de 2017 y requieren ser actualizados.

\section{4.- Propuesta}

\section{1.- Diseño de indicadores de cumplimiento (gestión y eficacia)}

Los indicadores aplicables a las variables identificadas en el proceso deben ser sencillos, contar con objetivos y de manejo fácil para su cometido. De estos se proponen indicadores de eficacia, el cual permite medir el nivel en el que se ejecutan las actividades planificadas y se logran los resultados deseados e indicadores, e indicadores de gestión, son aquellos que se emplean para contralar las operaciones, prevenir desviaciones que impidan el cumplimiento de objetivos 0 de metas.

\subsection{1.- Determinación de Indicadores de cumplimiento}

\section{Figura 3}

Indicadores de Gestión propuesto para el proceso de planificación.

\section{Indicadores de Eficacia - Proceso Planificación}

\section{Ahorro por Subasta Inversa Electrónica (SIE) \\ Eficacia en la contratación}

\section{Compras Imprevistas}

\section{Clasificador Central de Productos (CPCs)}

Cumplidos Plan Anual de Contracción PAC (\$)

Cumplimiento Planificación Plan Anual de Contracción (PAC) (\$)
Montos ahorrados por las entidades contratantes en los procesos realizados por SIE

Procesos no concretados en su contratación, en función del monto

Uso de compras imprevistas no consideras en el PAC

CPCs cumplidos PAC

Cumplimiento del Plan Anual de Compras
1 - (\$ total adjudicado SIE / $\$$ total presupuesto SIE).

1 - (\$ procesos desiertos + cancelados / $\$$ total presupuesto publicados)

1 - (\$ Ínfimas cuantiás / \$ total PAC (acumulado))

\$ CPCs cumplidos PAC / \$ total PAC Final

\$ Total Adjudicado / \$ PAC (anual) 
Indicadores de Gestión - Proceso Planificación

\begin{tabular}{|c|c|c|}
\hline Efectividad de Procesos & $\begin{array}{l}\text { Procesos } \\
\text { realizados }\end{array}$ & $\begin{array}{l}1 \text { - (Números de procesos } \\
\text { desiertos / Números total } \\
\text { publicados) }\end{array}$ \\
\hline $\begin{array}{l}\text { Cumplimiento del Plan } \\
\text { Anual de Compras (PAC) } \\
\text { por Clasificador Central } \\
\text { de Productos (CPC) }\end{array}$ & $\begin{array}{l}\text { Cumplimiento del plan anual } \\
\text { de compras por CPC }\end{array}$ & $\begin{array}{l}\text { Números CPCs cumplidos } \\
\text { PAC / Números CPCs } \\
\text { total PAC }\end{array}$ \\
\hline $\begin{array}{c}\text { Tiempos Fases } \\
\text { Precontractuales (Menor } \\
\text { cuantía Bienes y Servicios) }\end{array}$ & $\begin{array}{l}\text { Fecha efectiva de } \\
\text { adjudicación versus Fecha } \\
\text { estimada de adjudicación }\end{array}$ & $\begin{array}{l}\text { Números días planificados } \\
\text { para proceso } \\
\text { / Números días ejecutados }\end{array}$ \\
\hline $\begin{array}{c}\text { Tiempos Fases } \\
\text { Precontractuales Subasta } \\
\text { Inversa Electrónica (SIE) }\end{array}$ & $\begin{array}{l}\text { Fecha efectiva de } \\
\text { adjudicación versus Fecha } \\
\text { estimada de adjudicación }\end{array}$ & $\begin{array}{l}\text { Números días planificados } \\
\text { para proceso } \\
\text { / Números días ejecutados }\end{array}$ \\
\hline
\end{tabular}

Figura 1

Indicadores de eficacia propuesto para el proceso de planificación.

\section{Indicadores de Gestión - Prácticas de Contratación}

Competitividad en procedimientos de contratación por Subasta Inversa Electrónica (SIE)

\section{Contrataciones concursables} (Números procesos)

\section{Tiempos Fases}

Precontractuales (Menor Cuantía Bienes y Servicios)

\section{Calificación de}

Proveedores en Subasta Inversa Electrónica (SIE)

Procesos Competitivos en Subasta Inversa Electrónica (SIE)
Uso de puja en el procedimiento de SIE en función de número de procesos

Uso de herramienta de publicación con relación al resto de procedimientos

\section{Tiempos en fases} contractuales a discreción de la entidad en el procedimiento de Menor Cuantía

Calificaciones en procesos por puja

Procesos con participación múltiple de proveedores
Números procesos por puja /

Total procesos SIE

1 - (Números procesos por herramienta

de publicación / total procesos adjudicados)

Tiempos promedios / Tiempo promedio general

Proveedores calificados / Proveedores oferentes

Números procesos con tres o más proveedores calificados / Total procesos 


\section{Figura 5}

Indicadores de gestión propuesto para las prácticas de contratación.

\section{Indicadores de Eficacia - Prácticas de Contratación}

\begin{tabular}{|c|c|c|}
\hline $\begin{array}{c}\text { Competitividad en } \\
\text { procedimientos de } \\
\text { contratación por Subasta } \\
\text { Inversa Electrónica (SIE) } \\
\text { (monto adjudicado) }\end{array}$ & $\begin{array}{l}\text { Uso de puja en el } \\
\text { procedimiento } \text { SIE } \\
\text { función del monto }\end{array}$ & $\$$ por puja / $\$$ total SIE \\
\hline $\begin{array}{c}\text { Contrataciones } \\
\text { concursables (monto } \\
\text { adjudicado) }\end{array}$ & $\begin{array}{l}\text { Uso de herramienta de } \\
\text { publicación con relación al } \\
\text { resto de procedimientos en } \\
\text { función del monto }\end{array}$ & $\begin{array}{l}1 \text { - (\$ publicación / Total } \\
\text { monto adjudicado })\end{array}$ \\
\hline $\begin{array}{c}\text { Contrataciones por } \\
\text { Infimas }\end{array}$ & $\begin{array}{l}\text { Contrataciones imprevistas } \\
\text { en relación a lo adjudicado } \\
\text { por los regímenes de } \\
\text { contratación }\end{array}$ & $\begin{array}{l}\$ \quad \text { ínfimas } / \text { \$ } \$ \\
\text { adjudicado + ínfimas }\end{array}$ \\
\hline
\end{tabular}

Figura 6 Indicadores de eficacia propuesto para las prácticas de contratación.

\section{5.- CONCLUSIONES}

El levantamiento de la información ha permitido generar un enfoque sobre gestión por procesos que aplican los Direcciones Distritales de Salud, para el proceso de contratación pública, datos necesarios para realizar un diagnóstico de su situación actual:

- Se determinó que la cadena de valor permite establecer actividades que se desarrollan y son las bases para establecer mejoras en los procesos, como en el caso de contratación pública.

- $\quad$ Se estableció una matriz de causas y efectos, permitiendo visualizar los problemas principales que han incidido en el proceso de contratación pública.

- Se establecieron acciones correctivas que permitirán mitigar los problemas existentes y garantizar

- Se establecieron nuevos indicadores de cumplimiento que garantice la aplicación de las leyes, normas, entre otras que direccional en buen funcionamiento del proceso de contratación pública.

\section{6.- RECOMENDACIONES}

- Las Direcciones Distritales de Salud al aplicar las acciones correctivas permitirán alcanzar los objetivos establecidos para el proceso de contratación pública. 
- La incorporación de los indicadores de cumplimiento propuestos permitirá mantener una apropiada gestión por procesos, permitiendo que dichas métricas señalen el cumplimiento o incumplimiento de los procesos relacionados a la gestión de compras públicas.

- Las Direcciones Distritales de Salud deberán supervisar constantemente las mejoras planteadas al fin de mantener una apropiada gestión por procesos.

- $\quad$ Se recomienda la evaluación y mejora de los indicadores por lo menos una vez al año para mantener indicadores de cumplimiento óptimos para el proceso de contratación pública.

\section{7.- BIBLIOGRAFÍA}

Altamirano, G. (30 de Enero de 2014). Compras públicas. Obtenido de http://gabrieladelourdes.blogspot.com/2014/01/httpwww.html

Beltrán, J. (2005). Indicadores de Gestión, Guía Práctica para Estructurar Acertadamente ésta Herramienta Clave para el Logro de la Competitividad (Segunda ed.).

Córdova, J. (31 de Mayo de 2014). Falta de Insumos en los Hospitales. Obtenido de https://www.lavoz.com.ar/cordoba/falta-insumos-hospitales

Dessler, G. (2009). Adminsitración de Recursos Humanos, (11 ava ed.). México: Pearson Educación.

Hernández, R., Fernández, C., \& Baptista, M. (2014). Metodología de la Investigación, (Sexta ed.). México D.F: Mc Graw Hill Education/ Interamericana Editores S.A.

Lerma. (2014). Metodología de la investigación: propuesta, anteproyecto y proyecto (4ta edición ed.). Bogotá, Colombia: Ecoe Ediciones.

Organización Mundial del Comercio. (14 de Febrero de 2016). Panorama General de la Labor de la OMC en Materia de Contratación Pública. Obtenido de https://www.wto.org/spanish/tratop_s/gproc_s/overview_s.htm

Perez, J. (2004). Gestión por Procesos Cómo utilizar ISO 9001:2000 para mejorar la gestión de la organización. Madrid: Esic Editorial.

Sercop. (04 de 08 de 2008). Ley Orgánica del Sistema Nacional de Contratación Pública. Registro Oficial Suplemento 395 .

Soledispa, C. (13 de Marzo de 2016). Organización Panaméricana de Salud. Obtenido de https://www.paho.org/ecu/index.php?option=com_content\&view=article\&id=1118:enero24-2014\&Itemid=972 